\title{
Clave de identificación por ecolocación de 20 especies de murciélagos del suroeste de Perú
}

\author{
Key of the echolocation calls of 20 species of bats from southwestern Peru
}

1,a Joaquín A. Ugarte-Núñez

\section{ARTÍCULO ORIGINAL \\ ${ }^{1}$ Knight Piésold Consulting. Arequipa, Perú. E-mail: jugarte@knightpiesold.com}

aRCID: 0000-0001-6298-1818

Palabras clave: Desierto, parámetros bioacústicos, vertientes occidentales de los Andes.

Keywords: Desert, bioacoustic parameters, western slopes of the Andes.

Información adicional

Presentado: 27/09/2020

Aprobado: 28/11/2020

Este artículo cuenta con un preprint en el servicio de Scielo Preprints: https://doi.org/10.1590/SciELOPre prints. 1214

\section{RESUMEN}

Se presenta una clave de identificación por ecolocación y la descripción de los parámetros acústicos de 20 especies de murciélagos que ocupan hábitats similares de las regiones desérticas de la costa del Pacífico y vertientes occidentales de los Andes del Sur del Perú (departamentos de Arequipa, Moquegua y Tacna). La clave incluye la distribución de las especies en los principales hábitats y en los tres departamentos. Los parámetros de los llamados y la clave incluyen aspectos relacionados a su caracterización que permiten una fácil identificación en campo, como en gabinete. Se incluye un esquema a escala de los espectrogramas de los llamados de búsqueda. Se agrupan a las especies sobre la base de sus características taxonómicas, bioacústicas y de uso del hábitat de forrajeo. Esta clave será de ayuda para desarrollar acciones de monitoreo de la actividad, distribución y ecología para las 20 especies, o enfocar esfuerzos hacia las especies de distribución restringida (Lasiurus arequipae y Eumops chiribaya), los nuevos registros para esta región (Artibeus fraterculus y Eumops perotis), y aquellas amenazadas o raras (Tomopeas ravus, Myotis atacamensis, Amorphochilus schnablii, Mormopterus kalinowskii y Platalina genovensium).

\section{ABSTRACT}

An echolocation key and the description of acoustic parameters for 20 species of bats that occur in similar habitats of the Pacific coast and western slopes of the Andes of southern Peru (Arequipa, Moquegua and Tacna departments) was presented. The distribution of these species in habitats and every department was included in the key. The description of the calls and the key include aspects related to their characterization that allow easy the identification, both in the field and the Lab. The study also includes time and frequency parameters and scaled schematic drawings of the spectrograms. The species are clustered based on their taxonomy, acoustic features and foraging habitat use. This research will be useful to study the activity, distribution and ecology of these taxa, and additionally to concentrate efforts over short ranging species (i.e. Lasiurus arequipae and Eumops chiribaya), new records in the area (i.e. Artibeus fraterculus and Eumops perotis), and threatened or barely known bats (i.e. Tomopeas ravus, Myotis atacamensis, Amorphochilus schnablii, Mormopterus kalinowskii and Platalina genovensium). 


\section{INTRODUCCIÓN}

En Perú, los murciélagos representan la tercera parte de todos los mamíferos (Pacheco, 2018; Velazco, 2020); sin embargo, debido a su pequeño tamaño, hábitos nocturnos y refugios ocultos, este grupo se encuentra entre los más elusivos de los mamíferos, por lo que suelen ser subestimados en los inventarios, monitoreos u otros estudios (Obrist et al., 2004).

El monitoreo de murciélagos, como significativo indicador de biodiversidad, es cada vez más importante para entender y conocer los aspectos de su biología y ecología que nos permitan direccionar más eficazmente acciones de conservación y manejo, especialmente entender el impacto de origen humano sobre sus hábitats y poblaciones (Obrist et al., 2004; Fenton, 2013; Walters et al., 2013). Los estudios por medios acústicos cada vez van tomando mayor relevancia y uso, como un método complementario, alternativo y muchas veces más eficiente que los métodos tradicionales, que suelen representar deficientemente al grupo de los insectívoros aéreos (Kalko et al., 1996; O’Farrell \& Gannon 1999; Flaquer et al., 2007; Walters etal., 2013).

En Perú, los estudios acústicos son relativamente escasos, limitándose a nuevos registros y/o la descripción de los llamados o pulsos de ecolocación de algunas pocas especies: Platalina genovensium en un refugio por Malo de Molina et al. (2011), Myotis atacamensis en Lima por Pacheco et al. (2015) que probablemente sea Myotis bakeri de acuerdo con Moratelli et al. (2019), Amorphochilus schnablii por Falcao et al. (2015), Mormopterus kalinowskii por Flores et al. (2019), y Promops davisoni como primer registro para Chile por Ossa et al. (2018) incluyendo grabaciones realizadas en Tacna, al sur de Perú. Debido a la variación geográfica en los llamados que podría presentarse en algunas especies, así como el solapamiento que podría haber entre ellas (Orozco-Lugo, 2013; Ratcliffe \& Jakobsen, 2018; Russo et al., 2018), resultó importante obtener grabaciones específicas del suroeste de Perú (departamentos de Arequipa, Moquegua y Tacna).

Esta región del país comparte las mismas características ecológicas, como parte de la zona norte del desierto de Atacama y de la vertiente occidental de los Andes. De acuerdo con la Memoria Descriptiva del Mapa Nacional de Ecosistemas del Perú (MINAM, 2019), estos tres departamentos presentan los mismos ecosistemas.

La clave y caracterización acústica de las 20 especies hasta ahora incluidas en la quiropterofauna de esta región de Perú considera para Arequipa 20 especies, 16 incluidas por Pari et al. (2015), el nuevo registro de Eumops chiribaya por Medina et al. (2018), la descripción de Lasiurus arequipae por Málaga et al. (2020) y los registros de Artibeus fraterculus y Eumops perotis (Ugarte, por publicar). Para Moquegua, además de la descripción de E. chiribaya (Medina et al., 2014), se incluyen los registros acústicos de este trabajo, haciendo un total de 11 especies. Finalmente, para Tacna se considera un total de 11 especies, 7 registradas por Aragón \& Aguirre (2014) y Lanchipa \& Aragón (2018), a los que se suma el registro de Promops davisoni de Flores et al. (2015), el de Nyctinomops aurispinosus de Portugal-Zegarra et al. (2020), el registro de Lasiurus arequipae considerado como Lasiurus sp. en la tesis de Portugal (2018) y la adición de Nyctinomops macrotis con el registro acústico incluido en este trabajo.

\section{MATERIAL Y MÉTODOS}

El área que cubre la caracterización de los llamados de ecolocación corresponde a los departamentos de Arequipa, Moquegua y Tacna, ubicados en el suroeste de Perú. Esta región del país comparte las mismas características ecológicas, como parte de la zona norte del desierto de Atacama y de la vertiente occidental de los Andes. De acuerdo a la Memoria Descriptiva del Mapa Nacional de Ecosistemas del Perú (MINAM, 2019), estos tres departamentos presentan los siguientes ecosistemas, partiendo de la zona andina hacia la costa: pajonal de puna seca, bofedal, zona periglaciar y glaciar, matorral de puna seca, bosque relicto 
altoandino (Queñoal y otros), matorral andino, loma costera, desierto costero, humedal costero, además de lago y lagunas generalmente altoandinas y ríos provenientes de los deshielos de las zonas glaciares (en su mayoría) que desembocan en el océano Pacífico. Los tres departamentos, además presentan áreas intervenidas principalmente para uso agrícola, urbano, minero y para represas.

La caracterización de los llamados de ecolocación de las 20 especies de murciélagos se ha realizado sobre la base de grabaciones obtenidas en los departamentos de Arequipa, Moquegua y Tacna. La obtención de los llamados de referencia en fase de búsqueda se hizo sobre la base de trabajo de campo realizado entre junio del 2015 y febrero del 2020. Para la mayoría de las especies (15) se realizó la captura de individuos (permisos: RDG $\mathrm{N}^{\circ}$ 0114-2015-SERFOR-DGGSPFFS y RDG $\mathrm{N}^{\circ}$ 0065-2018-SERFOR-DGGSPFFS) usando redes de niebla (mist nets), para luego identificarlos hasta el nivel de especies en base a descripciones y claves (Ortiz de La Puente, 1951; Gregorin \& Taddei, 2002; Gardner, 2007; Diaz et al., 2016).

La grabación de los llamados se desarrolló cuando los individuos eran liberados en el lugar de la captura, luego de asegurarse de que no había otros individuos de la misma u otras especies pasando, para evitar falsas referencias. Las grabaciones se desarrollaron usando dos equipos Echo Meter 3 (Wildlife Acoustic) y Anabat Walkabout (Titley Scientific), ambos con una resolución de espectro completo (full spectrum) de 16bit, con un cociente de muestreo de $384 \mathrm{kHz}$, configurados a 10 segundos. Posteriormente, se realizaron grabaciones en vuelo libre en los mismos lugares donde se identificaron, liberaron y grabaron los individuos capturados, con el fin de validar el llamado de referencia grabado por liberación (Kunz \& Parsons, 2009; Estrada-Villegas, 2013).

Las especies que no pudieron ser capturadas fueron caracterizadas acústicamente por métodos combinados. De esta manera, Eumops chiribaya fue grabada en el lugar exacto a los pocos días de su captura en Arequipa (com. pers. Brian Málaga, 2017), confirmando que se trataba de la especie por lo característico del llamado para el género (Simmons \& Stein, 1980; Fenton, 1981; Bogdanowicz et al., 1999; Jung et al., 2014; AriasAguilar et al., 2018; López-Baucells et al., 2018) y porque los llamados no se parecían a los de ninguna otra especie caracterizada acústicamente antes en el suroeste de Perú. Para el caso de Eumops perotis, Nyctinomops aurispinosus, $N$. macrotis y Tomopeas ravus, su identificación se realizó por comparación con la bibliografía disponible (Pierson \& Rainey, 1998; Corbett et al., 2008; Collen, 2012; Jung et al., 2014; Arias-Aguilar et al., 2018; López-Baucells et al., 2018, com pers. Pacheco, 2020).

El análisis se hizo con el Software Raven Pro 1.5 (Center for Conservation Bioacoustics, Cornell Lab., 2014), para definir los principales parámetros acústicos de los llamados de búsqueda: frecuencia pico (PF en $\mathrm{kHz}$ ), frecuencia mínima ( $\mathrm{LF}$ en $\mathrm{kHz}$ ), frecuencia máxima ( $\mathrm{HF}$ en $\mathrm{kHz}$ ), el ancho de banda (BW en $\mathrm{kHz}$ ), duración de la llamada (CD en ms) e inter-pulso (IP en ms).

\section{RESULTADOS}

Los llamados de ecolocación de las 20 especies de murciélagos registradas para el suroeste de Perú presentan características de frecuencia y tiempo que permiten discriminarlas entre sí, en especial por la frecuencia pico, el ancho de banda y la duración. Estos parámetros incluidos en la clave corresponden a la fase de búsqueda usada con mayor frecuencia por las especies grabadas en los tres departamentos. 
Tabla 1

Clave de llamados de ecolocación de 20 especies del suroeste peruano

1a. Principalmente FM con un ancho de banda mayor a $20 \mathrm{kHz}$. 2

1b. Principalmente FQC, siempre un ancho de banda menor a $20 \mathrm{kHz} . \quad 11$ Pulsos fuertes en intensidad.

\begin{tabular}{lll}
\hline 2a. & Principalmente un solo componente, sin armónicos. & 3 \\
\hline 2b. & Dos o tres armónicos. Baja intensidad en los pulsos. & 7 \\
\hline
\end{tabular}

3a. Frecuencia pico mayor a $90 \mathrm{kHz}$ con un ancho de banda mayor a 60 Amorphochilus $\mathrm{kHz}$. Baja intensidad en los pulsos. Toda la costa, valles y desierto schnablii en Arequipa y Tacna. Ocasionalmente hasta $2800 \mathrm{~m}$ de altitud en Arequipa.

3b. Frecuencia pico menor a $90 \mathrm{kHz}$ con un ancho de banda menor a 4 $60 \mathrm{kHz}$. Pulsos fuertes en intensidad.

4a. Frecuencia pico mayor a $50 \mathrm{kHz}$, algunas veces supera los $60 \mathrm{kHz}, \quad$ Myotis generalmente alrededor de los $57 \mathrm{kHz}$. Diversos ambientes atacamenis naturales y antrópicos hasta $3500 \mathrm{~m}$ de altitud en los tres departamentos.

4b. $\quad$ Frecuencia pico menor a $50 \mathrm{kHz} \quad 5$

5a. FM descendente inicial y final con la porción media hacia una FQC Lasiurus leve. Llamado completo con forma de "S" muy extendida arequipae verticalmente. Frecuencia pico alrededor de $35 \mathrm{kHz}$ y una duración menor a $4 \mathrm{~ms}$. En valles costeros y de la vertiente occidental en Arequipa posiblemente hasta $3000 \mathrm{~m}$ de altitud. Costa de Tacna.

5b. $\quad$ FM descendente inicial con un componente de FQC suavizado al 6 final (frecuencia mínima).

6a. Frecuencia pico alrededor de $42 \mathrm{kHz}$ (desde 39 hasta $48 \quad$ Myotis oxyotus ocasionalmente). En valles costeros y de la vertiente occidental de Arequipa hasta $3300 \mathrm{~m}$ de altitud, generalmente cerca de cuerpos de agua.

6b. Frecuencia pico alrededor de $32 \mathrm{kHz}$ en búsqueda de insectos Histiotus voladores. Presenta llamados con armónicos cerca de vegetación, montanus con una frecuencia pico menor a $20 \mathrm{kHz}$ en busca de invertebrados perchados en la vegetación. Ambientes naturales y antrópicos desde el nivel del mar hasta más de $5000 \mathrm{~m}$ en los tres departamentos.

\begin{tabular}{lll}
\hline 7a. & Armónicos principalmente de FM sin solaparse significativamente. & 8
\end{tabular} Ancho de banda del llamado mayor a $55 \mathrm{kHz}$.

7b. Armónicos principalmente de FM que se solapan 9 significativamente. Ancho de banda del llamado menor a $55 \mathrm{kHz}$.

8a. Frecuencia pico alrededor de los $85 \mathrm{kHz}$. Áreas de cactáceas Platalina columnares principalmente desde cerca del nivel del mar en lomas genovensium costeras hasta los casi $3000 \mathrm{~m}$ de altitud en los tres departamentos.

8b. Frecuencia pico muy variable, generalmente alrededor de los $69 \quad$ Glossophaga $\mathrm{kHz}$, ocasionalmente baja a $60 \mathrm{kHz}$ o sube a más de $105 \mathrm{kHz}$. En soricina valles costeros y andinos de Arequipa hasta $\operatorname{los} 2200 \mathrm{~m}$ de altitud.

9a. Un componente inicial alrededor de los $40 \mathrm{kHz}$ con un segmento Desmodus corto de FQC y luego un descendente de FM. La frecuencia pico rotundus está alrededor de los 70 a $75 \mathrm{kHz}$ en el segundo armónico. Una secuencia se presenta en grupos de dos a cuatro llamados. Algunos valles costeros y zonas litorales con mamíferos marinos y ganado en Arequipa y Tacna.

9b. Sin ningún componente de FQC inicial, fuertes componentes de $\mathrm{FM}$, a veces con una leve FQC. 
10a. Frecuencia pico alrededor de $68 \mathrm{kHz}$ en el segundo armónico, a Sturnira cf veces alrededor de $87 \mathrm{kHz}$ en el tercero. En valles de la parte baja de la vertiente occidental en el norte de Arequipa, desde 1000 hasta bogotensis $1900 \mathrm{~m}$ de altitud.

10b. Frecuencia pico alrededor de $60 \mathrm{kHz}$. Ancho de banda menor a otros Phyllostomidae. Solamente en valles costeros del norte de Arequipa.

\begin{tabular}{llr}
\hline 11a. & Frecuencia pico mayor a 30 kHz. & 12 \\
\hline 11b. & Frecuencia pico menor a 30 kHz. & 15 \\
\hline 12a. & $\begin{array}{l}\text { Dos o más pulsos intercalados en un mismo pase o secuencia, } \\
\text { principalmente convexos. }\end{array}$ & 14 \\
\hline 12b. & Un solo tipo de pulso por pase o secuencia, nunca convexos. & $\begin{array}{l}\text { Molossus } \\
\text { molossus }\end{array}$ \\
\hline 13a. & $\begin{array}{l}\text { Pulso en FQC convexo descendente con un componente de FM } \\
\text { ascendente inicial corto. Intercala llamados entre 32 y 45 kHz } \\
\text { (frecuentemente a 35 y a 41 kHz). Duración entre 9 y 11 ms. Muy } \\
\text { raro en valles costeros del norte de Arequipa. }\end{array}$ & $\begin{array}{c}\text { Promops } \\
\text { davisoni }\end{array}$ \\
\hline 13b. & $\begin{array}{l}\text { Llamado más común es un pulso con un corto componente FM al } \\
\text { inicio seguido por uno FQC convexo ascendente, entre 32 y 34 kHz } \\
\text { de frecuencia pico. Intercala ocasionalmente con un segundo } \\
\\
\text { llamado de FM descendente con un componente principal FQC } \\
\text { alrededor de 38 kHz de frecuencia pico, con un ancho de banda } \\
\text { mayor. Ampliamente distribuido en desierto, valles costeros y } \\
\text { zonas urbanas hasta los 2700 m de altitud en los tres } \\
\text { departamentos. }\end{array}$ \\
\hline
\end{tabular}

14a. FQC descendente de corta duración ( $\sim 5 \mathrm{~ms})$ con una frecuencia pico alrededor de los $43 \mathrm{kHz}$. En valles costeros y quebradas secas de Arequipa.

14b. Llamado más común es FQC con un componente FM muy corto inicial ascendente con un pico a $34 \mathrm{kHz}$ (puede llegar Tomopeas ravus Artibeus fraterculus ocasionalmente a $37 \mathrm{kHz}$ ) con una duración de $10 \mathrm{~ms}$. Un segundo

Mormopterus kalinowskii tipo de llamado es FM descendente con un componente principal FQC alrededor de $42 \mathrm{kHz}$ de frecuencia pico con un ancho de banda y duración mayores. En todo tipo de ambientes naturales y antrópicos desde el nivel del mar a más de $2400 \mathrm{~m}$ en los tres departamentos.

\begin{tabular}{llr}
\hline 15a. & Frecuencia pico por debajo de 18 kHz & 16 \\
\hline 15b. & Frecuencia pico sobre 18 kHz & 18 \\
\hline 16a. & $\begin{array}{l}\text { Frecuencia pico a 17 kHz en dos tipos de pulsos (FMd-FQC y } \\
\text { FQC) con una duración de 14 ms aproximadamente. En desierto, } \\
\text { valles costeros y vertientes occidentales hasta 2300 m en los tres } \\
\text { departamentos. }\end{array}$ & $\begin{array}{r}\text { Nyctinomops } \\
\text { macrotis }\end{array}$ \\
\hline 16b. & Frecuencia pico por debajo de 17 kHz. & 17 \\
\hline 17a. & $\begin{array}{l}\text { Frecuencia pico entre 15 y 16 kHz, con un ligero componente } \\
\text { inicial FM descendente seguido por uno de FQC (típico del género) } \\
\text { con una duración de 11 a 14 ms. Valles costeros y quebradas secas } \\
\text { de Arequipa y Moquegua. }\end{array}$ & $\begin{array}{l}\text { Eumops } \\
\text { chibaya }\end{array}$ \\
\hline 17b. & $\begin{array}{l}\text { Frecuencia pico a 11 kHz con una duración mayor a 20 ms } \\
\text { generalmente. Solamente en valles costeros y quebradas del norte } \\
\text { de Arequipa. }\end{array}$ & Eumops perotis \\
\hline 18a. & $\begin{array}{l}\text { Frecuencia pico generalmente sobre 24 hasta 26 kHz en la misma } \\
\text { secuencia o pase en su forma de FQC. Puede pasar los 30 kHz } \\
\text { incrementando su ancho de banda en llamados de tipo FMd-FQC. }\end{array}$ & Tadarida \\
& $\begin{array}{l}\text { Los pulsos de FMd-FQC como de FQC, no se intercalan } \\
\text { regularmente. Ampliamente distribuido en todo tipo de ambientes }\end{array}$ \\
&
\end{tabular}


naturales y antrópicos hasta los $3660 \mathrm{~m}$ de altitud en los tres departamentos.

18b. Frecuencia pico por debajo de $24 \mathrm{kHz}$.

19a. Frecuencia pico de 18 a $20 \mathrm{kHz}$ de un llamado FQC típico con una duración de $13 \mathrm{~ms}$. También presenta pulsos FMd-FQC a $20 \mathrm{kHz}$. Valles costeros y zonas desérticas de los tres departamentos.

19b. Frecuencia pico de 22 a $23 \mathrm{kHz}$ de un llamado de FQC principalmente con un pequeño componente FM inicial con una duración de $9 \mathrm{~ms}$ en promedio. Puede llegar a solaparse con $T$. brasiliensis cuando usa FMd-FQC de banda más ancha. Valles costeros y desierto en Arequipa y Moquegua.

FM: Frecuencia modulada; FC: Frecuencia constante; FQC: Frecuencia casi (quasi) constante; kHz: kilohertz; ms: milisegundos.

Los parámetros de frecuencia y tiempo de los pulsos son muy variables entre las especies y están relacionados con la familia a la que pertenecen. De esta manera, como se puede ver en la Tabla 2 (principales parámetros acústicos medidos) y en la Figura 1 (que grafica el espectrograma característico de la fase de búsqueda), existen dos grandes grupos de tipos de llamados o pulsos de ecolocación: 1) los de frecuencia modulada descendente (FMd) con un amplio ancho de banda y, 2) los de frecuencia quasi-constante (FQC) que tienen un ancho de banda mucho menor y ecolocalizan a frecuencias más bajas; cada grupo representado por familias características.

Las especies del primer gran grupo (FM de amplio ancho de banda), se dividen en tres familias, dos de ellas, los Vespertilionidae (4 especies) y Furipteridae (1 especie), son insectívoros aéreos y generalmente no presentan armónicos en sus pulsos, y la tercera familia es la de los Phyllostomidae de hábitos alimenticios más variados, que pueden diferenciarse en la forma de sus llamados, siendo Desmodus rotundus (hematófago) una especie con los pulsos de ecolocación más complejos por presentar un primer armónico con un segmento de FQC, los frugívoros (Artibeus fraterculus y Sturnira cf bogotensis) tienen armónicos que se solapan en gran parte de su rango de frecuencias y, los nectarívoros (Platalina genovensium y Glossophaga soricina) que casi no se solapan y tienen un ancho de banda mayor.

El segundo gran grupo (FQC con un ancho de banda mucho menor) pertenece íntegramente a la familia Molossidae. Todos son insectívoros aéreos, que a su vez se pueden subdividir en aquellas especies con el pulso convexo (Molossus molossus y Promops davisoni) y el resto de las especies (8) con pulso cóncavo o sin inflexión, llegando casi a frecuencia constante (FC).

\section{Tabla 2}

Parámetros acústicos principales de 20 especies de quirópteros del suroeste de Perú

\begin{tabular}{|c|c|c|c|c|c|c|c|c|}
\hline Especies\Parámetros & $\begin{array}{c}\text { PF } \\
(\mathbf{k H z})\end{array}$ & $\begin{array}{c}\mathbf{L F} \\
(\mathbf{k H z}) \\
\end{array}$ & $\begin{array}{c}\mathbf{H F} \\
(\mathbf{k H z})\end{array}$ & $\begin{array}{c}\text { BW } \\
(\mathbf{k H z})\end{array}$ & $\begin{array}{l}\text { Dur } \\
(\mathbf{m s})\end{array}$ & $\begin{array}{l}\text { IP } \\
(\mathbf{m s})\end{array}$ & $\mathbf{N}$ & Tipo \\
\hline \multirow{2}{*}{ Artibeus fraterculus } & 61.22 & 46.71 & 91.60 & 44.89 & 2.98 & 78.65 & \multirow{2}{*}{116} & \multirow{2}{*}{$\begin{array}{c}\overline{\mathrm{FMd} /} \\
\mathrm{a}\end{array}$} \\
\hline & \pm 3.59 & \pm 4.40 & \pm 6.41 & \pm 7.60 & \pm 0.91 & \pm 28.75 & & \\
\hline \multirow{2}{*}{ Desmodus rotundus } & 72.92 & 33.28 & 85.93 & 52.65 & 4.73 & 29.47 & \multirow{2}{*}{113} & $\mathrm{FMd} /$ \\
\hline & \pm 3.95 & \pm 1.73 & \pm 9.51 & \pm 9.17 & \pm 1.46 & \pm 18.12 & & $\mathrm{a}$ \\
\hline \multirow{2}{*}{ Glossophaga soricina } & 67.66 & 44.84 & $\mathbf{1 3 4 . 8 0}$ & 89.96 & 1.19 & 30.23 & \multirow{2}{*}{123} & FMd/ \\
\hline & \pm 4.90 & \pm 6.22 & \pm 7.96 & \pm 8.99 & \pm 0.39 & \pm 19.46 & & $a$ \\
\hline \multirow{2}{*}{ Platalina genovensium } & 84.97 & 44.89 & 116.12 & 71.23 & 1.29 & 61.46 & \multirow{2}{*}{447} & FMd/ \\
\hline & +2.79 & +2.01 & +2.92 & +3.14 & \pm 0.46 & +47.45 & & a \\
\hline \multirow{2}{*}{$\begin{array}{l}\begin{array}{l}\text { Sturnira cf bogotensis }\left(2^{\circ}\right. \\
\text { arm) }\end{array} \\
\text { arm }\end{array}$} & $\overline{68.55}$ & $\overline{51.88}$ & $\mathbf{1 1 0 . 7 3}$ & $\overline{58.85}$ & 2.08 & 71.15 & \multirow{2}{*}{125} & FMd/ \\
\hline & \pm 2.71 & +4.82 & \pm 6.62 & \pm 8.70 & \pm 0.55 & \pm 19.97 & & $\mathrm{a}$ \\
\hline \multirow{2}{*}{ S. cf bogotensis ( $\left(3^{\circ} \mathrm{arm}\right)$} & 87.13 & - & - & - & - & - & \multirow{2}{*}{124} & \\
\hline & +4.21 & -- & -- & -- & -- & -- & & \\
\hline
\end{tabular}




\begin{tabular}{|c|c|c|c|c|c|c|c|c|}
\hline Especies\Parámetros & $\begin{array}{c}\text { PF } \\
(\mathbf{k H z})\end{array}$ & $\begin{array}{c}\mathbf{L F} \\
(\mathbf{k H z})\end{array}$ & $\begin{array}{c}\text { HF } \\
(\mathbf{k H z})\end{array}$ & $\begin{array}{c}\text { BW } \\
(\mathbf{k H z})\end{array}$ & $\begin{array}{l}\text { Dur } \\
(\mathrm{ms})\end{array}$ & $\begin{array}{c}\text { IP } \\
(\mathrm{ms})\end{array}$ & $\mathbf{N}$ & Tipo \\
\hline \multirow{2}{*}{ Amorphochilus schnablii } & 99.70 & 78.75 & 138.92 & 60.17 & 2.58 & 32.06 & \multirow{2}{*}{591} & \multirow{2}{*}{ FMd } \\
\hline & +4.87 & +4.46 & \pm 7.84 & \pm 10.59 & \pm 0.69 & \pm 10.28 & & \\
\hline \multirow{2}{*}{ Histiotus montanus } & 31.36 & 22.18 & 59.67 & 37.49 & 6.10 & 132.10 & \multirow{2}{*}{302} & \multirow{2}{*}{ FMd } \\
\hline & \pm 2.29 & +1.16 & +3.52 & +3.55 & \pm 0.61 & +17.63 & & \\
\hline \multirow{2}{*}{ Lasiurus arequipae } & 35.39 & 19.27 & 75.56 & 56.29 & 3.97 & 91.31 & \multirow{2}{*}{74} & \multirow{2}{*}{ FMd } \\
\hline & \pm 3.44 & \pm 2.41 & \pm 11.78 & \pm 12.78 & \pm 1.23 & \pm 46.69 & & \\
\hline \multirow{2}{*}{ Myotis atacamensis } & $\mathbf{5 7 . 8 1}$ & 51.40 & 90.09 & 38.69 & 2.51 & 64.04 & \multirow{2}{*}{$\begin{array}{c}310 \\
0\end{array}$} & \multirow{2}{*}{ FMd } \\
\hline & +5.04 & +5.61 & \pm 11.88 & \pm 12.49 & \pm 0.99 & \pm 14.29 & & \\
\hline \multirow{2}{*}{ Myotis oxyotus } & 41.48 & 39.30 & 56.51 & 33.35 & 3.21 & 107.77 & \multirow{2}{*}{325} & \multirow{2}{*}{ FMd } \\
\hline & \pm 3.45 & +3.09 & \pm 9.17 & \pm 8.06 & \pm 1.41 & +58.33 & & \\
\hline \multirow{2}{*}{ Eumops perotis } & 11.50 & 8.60 & 14.24 & 5.63 & 22.33 & 98.00 & & \multirow{2}{*}{$\begin{array}{l}\text { FMd- } \\
\text { FQC }\end{array}$} \\
\hline & \pm 0.46 & +1.10 & +1.29 & \pm 2.26 & +4.73 & \pm 5.66 & & \\
\hline \multirow{2}{*}{ Eumops chiribaya } & 15.75 & 15.24 & 20.10 & 4.86 & 11.85 & 307.32 & \multirow{2}{*}{101} & \multirow{2}{*}{$\begin{array}{l}\text { FMd- } \\
\text { FQC }\end{array}$} \\
\hline & \pm 1.09 & \pm 0.59 & \pm 2.59 & \pm 2.38 & \pm 2.60 & \pm 123.29 & & \\
\hline \multirow{2}{*}{ Molossus molossus (bajo) } & 34.94 & 31.66 & 36.95 & 5.29 & 10.46 & 97.90 & \multirow{2}{*}{80} & \multirow{2}{*}{ FQCd } \\
\hline & +1.90 & \pm 2.24 & \pm 2.22 & \pm 1.34 & \pm 2.38 & +47.33 & & \\
\hline \multirow{2}{*}{ M. molossus (alto) } & 41.95 & 38.68 & 44.08 & 5.40 & 9.50 & 97.90 & \multirow{2}{*}{60} & \multirow{2}{*}{ FQCd } \\
\hline & \pm 1.94 & \pm 1.92 & \pm 2.33 & \pm 1.60 & \pm 2.26 & \pm 47.33 & & \\
\hline Marmontorus kalinouskii & 34.75 & 33.73 & 35.32 & 1.49 & 9.73 & 184.98 & 330 & (FM)- \\
\hline 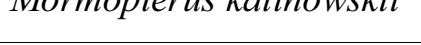 & \pm 2.68 & \pm 2.63 & \pm 2.96 & \pm 0.78 & \pm 2.18 & +92.24 & (3J) & $\mathrm{FQCd}$ \\
\hline$c t$ & 18.79 & 17.95 & 19.31 & 1.38 & 13.40 & 430.62 & 71 & (FM)- \\
\hline sus & \pm 1.43 & \pm 1.55 & \pm 1.62 & \pm 1.13 & \pm 3.32 & \pm 88.25 & 11 & FQCd \\
\hline$m$ & 22.91 & 21.78 & 23.49 & 1.70 & 9.40 & 304.01 & & (FM)- \\
\hline atıcaudatus & \pm 1.17 & \pm 1.09 & \pm 1.48 & \pm 0.76 & \pm 2.00 & \pm 174.00 & 63 & $\mathrm{FQCd}$ \\
\hline & 17.43 & 15.12 & 18.95 & 3.83 & 14.60 & 514.12 & & (FM)- \\
\hline Dits & \pm 0.66 & \pm 0.71 & \pm 0.94 & \pm 0.97 & \pm 1.96 & \pm 151.36 & 92 & FQCd \\
\hline Promops davisoni & 31.96 & 31.08 & 32.24 & 1.15 & 10.18 & 190.52 & 607 & FMa- \\
\hline & \pm 0.50 & \pm 0.61 & \pm 0.54 & \pm 0.36 & \pm 1.46 & \pm 112.47 & & $\mathrm{FQCa}$ \\
\hline ) & 38.47 & 35.81 & 46.13 & 8.72 & 10.06 & 95.59 & & FMd- \\
\hline ) & \pm 2.30 & \pm 4.12 & \pm 3.64 & \pm 3.45 & \pm 2.72 & \pm 27.72 & & FQCd \\
\hline & 25.16 & 23.33 & $\overline{31.22}$ & 7.88 & 10.03 & $\mathbf{2 3 0 . 8 7}$ & & (FM)- \\
\hline I adarla brastllensls & \pm 0.95 & +2.99 & +3.93 & \pm 4.80 & \pm 2.21 & +95.43 & 421 & FQCd \\
\hline & 43.40 & 41.56 & 45.71 & 4.15 & 5.18 & 122.79 & 50 & \\
\hline 1omopeas ravus & \pm 0.90 & +1.62 & +1.36 & \pm 2.30 & \pm 1.09 & +22.37 & 50 & \\
\hline
\end{tabular}

Nota: La media se muestra en negrita y debajo, su desviación estándar. PF (kHz): Frecuencia pico en kilohertz; LF (kHz): Frecuencia mínima; HF (kHz): Frecuencia máxima; BW (kHz): Ancho de banda; Dur (ms): Duración del llamado en milisegundos; IP (ms): Interpulso; N: Cantidad de pulsos medidos; FM: Frecuencia modulada; FQC: frecuencia casi constante; d: descendente; a: ascendente; /a: con armónicos. 


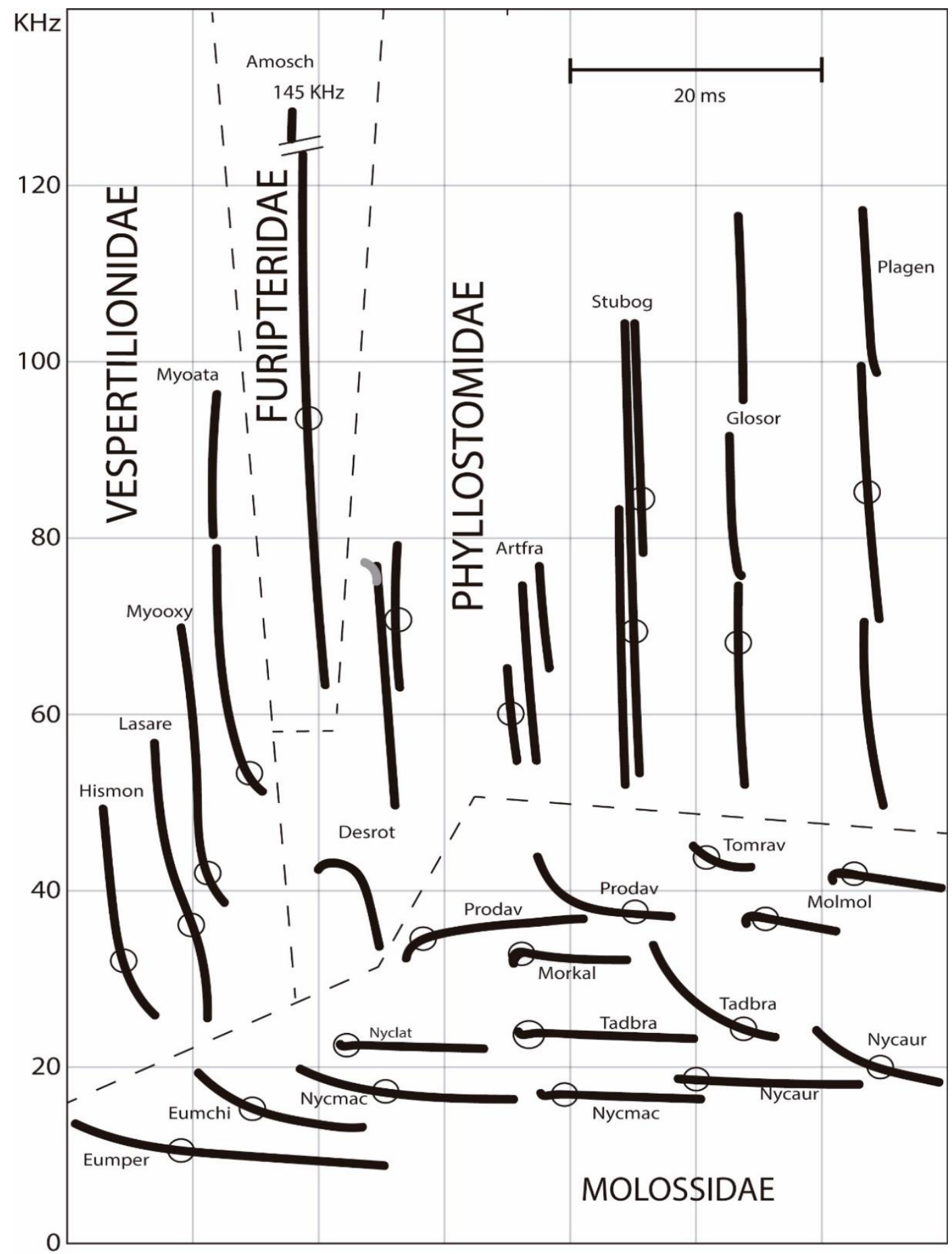

Figura 1. Espectrogramas esquematizados de los llamados de ecolocación de la fase de búsqueda de 20 especies de murciélagos del Suroeste de Perú.

Nota: El círculo muestra la frecuencia pico del llamado. Las líneas punteadas separan las familias. Especies: Hismon: Histiotus montanus; Lasare: Lasiurus arequipae; Myooxy: Myotis oxyotus; Myoata: Myotis atacamensis; Amosch: Amorphochilus schnablii; Desrot: Desmodus rotundus; Artfra: Artibeus fraterculus; Stubog: Sturnira cf bogotensis; Glosor: Glossophaga soricina; Plagen: Platalina genovensium; Eumper: Eumops perotis; Eumchi: Eumops chiribaya; Nycmac: Nyctinomops macrotis; Nycaur: Nyctinomops aurispinosus; Nyclat: Nyctinomops laticaudatus; Tadbra: Tadarida brasiliensis; Morkal: Mormopterus kalinowskii; Prodav: Promops davisoni; Molmol: Molossus molossus; y Tomrav: Tomopeas ravus. 


\section{DISCUSIÓN}

El diseño de los pulsos de ecolocación depende en gran medida de los hábitos alimenticios que determinan las características del espacio en el que los murciélagos buscan su alimento (Neuwelier, 2000; Schnitzler \& Kalko, 2001; Denzinger \& Schnitzler, 2013).

De esta manera, el grupo de murciélagos que cazan insectos en el aire dentro de la vegetación, sus bordes, en espacios abiertos y/o lagunas o ríos, incluye a especies de las familias Vespertilionidae y Furipteridae, que usan componentes de FM de banda ancha, los más adecuados para la localización y caracterización de sus objetivos presa (insectos voladores) en medio de un fondo de vegetación, que también debe ser detectado para evitar colisiones (Schnitzler \& Kalko, 2001).

Esta descripción FM de banda ancha es aplicable a aquellas especies que no necesariamente son insectívoras, pero que buscan su alimento en la vegetación, como son los Phyllostomidae, los que también usan pulsos o llamados de frecuencia modulada para diferenciar los objetos de fondo, pero, con una baja intensidad de los pulsos (su alimento no está en movimiento), además de complementar su búsqueda con la vista y el olfato (Kalko, 2004).

Es sí que, la clave, parámetros y esquema de los espectrogramas incluyen la caracterización de los pulsos de ecolocación de la familia Phyllostomidae, a pesar de ser un grupo que se considera que no es buen candidato para estudios acústicos (dificultad de registrarlos por sus llamados, por su baja intensidad y altas frecuencias); sin embargo, es posible que se puedan incluir en estudios acústicos en la medida que se conozcan más las variaciones intra e interespecíficas, especialmente la frecuencia pico, número de armónicos, intervalo entre pulsos, entre otros parámetros de los llamados de búsqueda (Kalko, 2004).

Asimismo, los Phyllostomidae de estas regiones áridas del país están representados por pocas especies, por lo que su discriminación en fase de búsqueda puede realizarse con relativa facilidad, ya que no existe solapamiento de sus frecuencias pico o la forma cómo se configuran sus armónicos, además que la estructura del pulso está relacionada con el tipo de alimentación. El uso de la acústica es útil en esta familia, para aspectos más dirigidos, como el monitoreo de la alimentación en plantas (cactus por ejemplo para $P$. genovensium) o el uso de refugios, ya que la baja intensidad de sus llamados solo permite un registro a distancias muy cortas $(\sim 1 \mathrm{~m})$.

El grupo con frecuencias y ancho de banda menores (Molossidae) incluye a especies insectívoras que forrajean en espacios abiertos, sobre la vegetación, donde las presas están dispersas y son de baja densidad, lo que requiere de pulsos de ecolocación diseñados para un rango de detección mayor sin tener que evitar obstáculos (no tienen vegetación de fondo), por lo que reducen significativamente el ancho de banda para concentrar la energía en un rango estrecho de frecuencias, aumentando considerablemente la duración del llamado (Neuwelier, 2000; Jung et al., 2014). Dentro de este grupo, aquellas especies que muestran un ancho de banda mayor o que intercalan sus llamados son los que generalmente pueden buscar su alimento cerca de la vegetación y el suelo, además del espacio aéreo (Gillam et al., 2009), por lo que son los que normalmente se registran bajo los métodos tradicionales (redes de niebla o trampas arpa) (e.g. P. davisoni, M. kalinowskii y Tadarida brasiliensis), lo que deja con muy pocos registros en el suroeste de Perú a especies de los géneros Nyctinomops o Eumops por ejemplo (Aragón \& Aguirre, 2014; Pari et al., 2015; Lanchipa \& Aragón, 2018), que terminan siendo submuestreados.

Finalmente, al usar los parámetros descritos en la Tabla 2, así como la clave, es importante considerar estos llamados como referenciales, debido a que la ecolocación en los murciélagos, como su principal sistema sensorial, puede tener algunas variaciones dependiendo de diversas circunstancias ecológicas y de comportamiento, incluso variando entre individuos de la misma especie, o cerca de sus presas, por el tamaño 
del área de vuelo, por la presencia de otras especies de murciélagos en el área, entre otros factores (Gillam et al., 2009). Por ejemplo, entre Nyctinomops laticaudatus y Tadarida brasiliensis puede haber solapamientos por tratarse de especies que alternan mucho las frecuencias pico de sus llamados, típico de algunos miembros de la familia, por su plasticidad (Jung et al., 2014).

\section{AGRADECIMIENTOS}

El autor agradece la asistencia en el campo de Joaquín y Adriana Ugarte Mosaurieta, de Hugo Zamora y Verónica Gómez. Asimismo, los valiosos aportes de Brock Fenton y Bruce Miller en el diseño final de la clave.

\section{REFERENCIAS}

Aragón, G., \& Aguirre, M. (2014). Distribución de murciélagos en la región de Tacna (Perú). Idesia, 32(1), 119-127.

Arias-Aguilar, A., Hintze, F., Aguiar, L., Rufray, V., Bernard, E., \& Ramos, M. (2018). Who's calling? Acoustic identification of Brazilian bats. Mamm Res, 63, 231-253. https://doi.org/10.1007/s13364018-0367-z

Bogdanowicz, W., Fenton, M., \& Daleszczyk, K. (1999). The relationships between echolocation calls, morphology and diet in insectivorous bats. Journal of Zoology, 247, 381 - 393. https://doi.org/10.1111/j.1469-7998.1999.tb01001.x.

Collen, A. (2012). The evolution of echolocation in bats: a comparative approach (Dissertation). University College London.

Corbett, R., Chambers, C., \& Herder, M. (2008). Roosts and activity areas of Nyctinomops macrotis in northern Arizona. Acta Chiropt, 10(2), 323-329

Denzinger, A., \& Schnitzler, H. (2013). Bat guilds, a concept to classify thehighly diverse foraging and echolocation behaviors ofmicrochiropteran bats. Front Physiol, 4, 164. https://doi.org/10.3389/fphys.2013.00164

Díaz, M., Solari, S., Aguirre, L., Aguiar, L., \& Barquez, R. (2016). Clave de identificación de los murciélagos de Sudamérica. Publicación Especial N² 2, PCMA (Programa de Conservación de los Murciélagos de Argentina).

Estrada-Villegas, S., Rodriguez, R., \& Barboza, K. (2013). Ecolocación: fundamentos, usos y equipos. Disponible en: http://www.relcomlatinoamerica.net/indez.php/bioacustica; 06, septiembre, 2020)

Falcão, F., Ugarte-Núñez, J. Faria, D., \& Caselli, C. (2015). Unravelling the calls of discrete hunters: Acoustic structure of echolocation calls of furipterid bats (Chiroptera, Furipteridae). Bioacoustics 24(2):175-183. https://doi.org/10.1080/09524622.2015.1017840

Fenton, M., \& Bell, G. (1981). Recognition of species of insectivorous bats by their echolocation calls. $J$. Mammlgy, 62, 233-242.

Fenton, M. (2013). Questions, ideas and tools: lessons from bat echolocation. Animal Behaviour, 85, 869879.

Flaquer, C., Torre, I., \& Arrizabalaga, A. (2007). Comparison of sampling methods for inventory of bat communities. Journal of Mammalogy, 88, 526-533.

Flores, M., Calizaya, G., Pacheco, V., \& Aragón, G. (2015). Distribution of Promops davisoni Thomas, 1921 (Chiroptera: Molossidae) in Peru with a new record and southward range extension. Check List, 11(2): 1.573.

Flores, M., Calizaya, G., Portugal-Zegarra, G., Aragón, G., Pacheco-Castillo, J., \& Rengifo. E. (2019). Contributions to the natural history of Mormopterus kalinowskii (Chiroptera: Molossidae) in the southwest of Peru Therya Advance. THERYA. https://doi.org/10. 10.12933/therya-19-753.

Gardner, A. (2007). Order Chiroptera. In: Mammals of South America. Volume I Marsupials, Xenarthrans, Shrews, and bats. University of Chicago Press. Chicago and London. United Kingdom. 
Gillam, E., McCracken, G., Westbrook, J., Lee, Y., Jensen, M., \& Balsley, B. (2009). Bats aloft: variability in echolocation call structure at high altitudes. Behav Ecol Sociobiol, 64, 69-79.

Gregorin, R., \&Taddei, V.A. (2002). Chave artificial para a identificação de molossídeos brasileiros (Mammalia, Chiroptera). Mastozoologia Neotropical 9(1), 13-32.

Jung, K., Molinari, J., \& Kalko, E. (2014). Driving Factors for the Evolution of Species-Specific Echolocation Call Design in New World Free-Tailed Bats (Molossidae). PLoS ONE 9(1): e85279. doi:10.1371/journal.pone.0085279

Kalko, E. (2004). Neotropical leaf-nosed bat (Phyllostomidae): "Whispering" bats as candidates for acoustic surveys? Pp. 63-69 in Bat echolocation research: tools, techniques and analysis (Brigham, M., E. K V. Kalko, G. Jones, S. Parsons, y H. J. G. A. Limpens, eds.). Bat Conservation International. Austin, EE.UU. 44 THERYA 4 (1):33-46

Kalko, E., Handley, C., \& Handley, D. (1996). Organization, diversity, and long term dynamics of a Neotropical bat community. in Long term studies in vertebrate communities (Cody, M., y J. Smallwood, eds.). Academic Press. Los Angeles, EE.UU.

Kunz, T., \& Parson, L. (2009). Ecological and behavioral methods for the study of bats. Baltimore: Johns Hopkins University Press.

Lanchipa, T., \& Aragón, G. (2018). Ensamble de murciélagos en el valle de Ite, región Tacna, Perú. Idesia, 36(1), 83-90.

López-Baucells, A., Rocha, R., Bobrowiec, P., Bernard, E., Palmeirim, J., \& Meyer. C. (2018). Field Guide to the Bats of the Amazon. Pelagic Publishing, Exeter, United Kingdom.

Málaga, B., Díaz, D., Arias S., \& Medina. C. (2020). Una especie nueva de Lasiurus (Chiroptera: Vespertilionidae) del suroeste de Perú. Revista Mexicana de Biodiversidad, 91, e913096. https://doi.org/10.22201/ib.20078706e.2020.91.3096

Malo de Molina, J., Velazco, S., Pacheco, V., \& Robledo, J. (2011). Análisis de las Vocalizaciones del Murciélago Longirrostro Peruano Platalina genovensium Thomas, 1928 (Chiroptera: Phyllostomidae). Revista Peruana de Biología, 18 (3), $311-18$. https://doi.org/10.15381/rpb.v18i3.443

Medina, C., Diaz, D., Málaga, B. Medina, Y., \& López, E. (2018). Short Communication: Second record of Eumops chiribaya (Chiroptera, Molossidae) in Peru. Biodiversitas, 19 (6). https://doi.org/10.13057/biodiv/d190601.

Medina, C., Gregorin, R., Zeballos, H., Zamora, H., \& Moras. L. (2014). A new species of Eumops (Chiroptera: Molossidae) from southwestern Peru. Zootaxa 3878, 19-36. https://doi.org/10.11646/zootaxa.3878.1.2.

MINAM. (2019). Mapa Nacional de Ecosistemas del Perú: Memoria Descriptiva. Ministerio del Ambiente de Perú.

Moratelli, R., Novaes, L., Carrión, C., \& Wilson., D. (2019). A New Species of Myotis (Chiroptera, Vespertilionidae) from Peru. Special Publications, Museum of Texas Tech University.

Neuweiler, G. (2000).The biology ofbats. NewYork: Oxford University.

Obrist, M., Boesch, R., \& Flückiger, P. (2004). Variability in echolocation call design of 26 Swiss bat species: consequences, limits and options for automated field identification with a synergetic pattern recognition approach. Mammalia, 68(4). https://doi.org/10.1515/mamm.2004.030

O'Farrell, M., \& Gannon, W. (1999). A comparison of acoustics versus capture techniques for the inventory of bats. Journal of Mammalogy 80, 24-30.

Orozco-Lugo, L., Guillén-Servent, A., Valenzuela-Galván D., \& Arita, H. (2013). Descripción de los pulsos de ecolocalización de once especies de murciélagos insectívoros aéreos de una selva baja caducifolia en Morelos, México. Therya, 4, 33-46.

Ortiz de la Puente, J. (1951). Estudio monográfico de los quirópteros de Lima y alrededores. Pub. Mus. de Hist. Nat., 7, 1-48. 
Ossa, G., Lilley, M., Ugarte-Núñez, J., Ruokolainen, L., K. Vilches, K., Valladares-Faúndez, P., \& Yung. V. (2018). First record of Promops davisoni (Thomas, 1921) (Chiroptera, molossidae) from Chile and a description of its echolocation calls. Mastozoología Neotropical, Mendoza.

Pacheco, V., Zevallos, A., Cervantes, K., Pacheco J., \& Salvador. J. (2015). Mamíferos del Refugio de Vida Silvestre Los Pantanos de Villa. Cientifica, 12 (1), 26-41.

Pacheco, V., Inche B., \& Wust. W. (2018). Mamíferos del Perú. Grupo La República.

Pari, A., Pino, K., Medina, C., López, E., \& Zeballos. H. (2015). Murciélagos de Arequipa, Historia Natural yConservación. Arequipa, Perú.

Pierson, E. \& Rainey. W. (1998). Distribution, Habitat Associations, Status, and Survey Methodologies for Three Molossid Bat Species (Eumops perotis, Nyctinomops femorosaccus, Nyctinomops macrotis) and the Vespertilionid (Euderma maculatum). Final Report Prepared for: California Department of Fish and Game Wildlife Management Division Bird and Mammal Conservation Program.

Portugal, G. (2018). Diversidad y distribución del orden Chiroptera en el valle de Sama, Tacna. Tesis para optar el título profesional de biólogo. Universidad Nacional Jorge Basadre Grohmann - Tacna, Perú.

Portugal-Zegarra, G., Flores-Quispe, M., Calizaya-Mamani, G., \& Aragón, G. (2020). New record of Nyctinomops aurispinosus with an update of its known distribution. Therya Notes 2020, 1 (1), 6776. https://doi.org/10.12933/therya_notes-20-16

Ratcliffe, J., \& Jakobsen, L. (2018). Don't believe the mike: behavioural, directional, and environmental impacts on recorded bat echolocation call measures. Canadian Journal of Zoology, 96 (4), 283-288. https://doi.org/10.1139/cjz-2017-0219

Russo, D., Ancillotto, L., \& Jones, G. (2018). Bats are still not birds in the digital era: echolocation call variation and why it matters for bat species identification. Canadian Journal of Zoology, 96, 63-78.

Schnitzler, H., \& Kalko E. (2001). Echolocationby Insect-Eating Bats. Bioscience, 51(7), 557-569.

Simmons, J., \& Stein, R. (1980). Acoustic imaging in bat sonar: Echolocation signals and the evolution of echolocation. J. Comp. Physiol. 135, 61-84. https://doi.org/10.1007/BF00660182.

Velazco, P. (2020). Murciélagos del Perú / Bats of Peru. http://www.paulvelazco.com/murcielagos_peru.html. Accessed on July 18, 2020.

Walters, C., Collen, A., Lucas, T., Mroz, K., Sayev, C., \& Jones, K. (2013). Challenges of using bioacoustics to globally monitor bats. In Bat Evolution, Ecology, and Conservation (eds R. A. Adams and S. C. Pedersen). New York: Springer. 